\title{
Planar and Plane Slope Number of Partial 2-Trees
}

\author{
William Lenhart ${ }^{1}$, Giuseppe Liotta ${ }^{2}$, \\ Debajyoti Mondal ${ }^{3}$, and Rahnuma Islam Nishat ${ }^{4}$ \\ ${ }^{1}$ Department of Computer Science, Williams College, MA, USA \\ ${ }^{2}$ Dipartimento di Ingegneria Elettronica e dell'Informazione, \\ Universita' degli Studi di Perugia, Italy \\ ${ }^{3}$ Department of Computer Science, University of Manitoba, Canada \\ ${ }^{4}$ Department of Computer Science, University of Victoria, Canada \\ lenhartecs.williams.edu, liotta@diei.unipg.it, \\ jyoti@cs.umanitoba.ca, rnishat@uvic.ca
}

\begin{abstract}
We prove tight bounds (up to a small multiplicative or additive constant) for the plane and the planar slope numbers of partial 2-trees of bounded degree. As a byproduct of our techniques, we answer a long standing question by Garg and Tamassia about the angular resolution of the planar straight-line drawings of series-parallel graphs of bounded degree.
\end{abstract}

\section{Introduction}

A drawing of a graph $G$ in $\mathbb{R}^{2}$ maps each vertex of $G$ to a point and each edge of $G$ to a Jordan arc such that an edge does not contain a vertex other than its endpoints, no edge crosses itself, edges do not meet tangentially, and edges sharing a common end-vertex do not cross each other. A planar graph is a graph that admits a planar drawing, i.e. a drawing such that no two edges intersect except at their common end-points. A plane graph is a planar graph together with a combinatorial embedding, i.e. a prescribed set of faces including a prescribed outer face. A plane drawing of a plane graph $G$ is a planar drawing that realizes the combinatorial embedding of $G$.

The slope number of a straight-line drawing $\Gamma$ of a planar graph $G$ is the number of distinct slopes of the edges of $\Gamma$. Every plane (planar) graph admits a plane (planar) straight-line drawing [1], i.e. a drawing where the edges are mapped to straight line segments. The planar slope number of $G$ is the smallest slope number over all planar straight-line drawings of $G$. If $G$ is a plane graph, the plane slope number of $G$ is the smallest slope number over all plane straight-line drawings of $G$.

The problem of computing drawings of planar graphs with maximum degree four, using only horizontal and vertical slopes, has long been studied in graph drawing through the research on orthogonal and rectilinear graph drawing (see, e.g., [1]). In a seminal paper, Dujmović et al. [2] extend this study to non-orthogonal slopes, and give tight upper and lower bounds (expressed as functions of the number $n$ of vertices) on the plane slope numbers of several graph families including plane 3 -trees and plane 3-connected graphs. They also ask whether the plane slope number of a plane graph of maximum degree $\Delta$ can be bounded by a function $f(\Delta)$. Keszegh et al. [7] answer the question affirmatively proving that, for a suitable constant $c$, the plane slope number of a plane

S. Wismath and A. Wolff (Eds.): GD 2013, LNCS 8242, pp. 412 423, 2013.

(C) Springer International Publishing Switzerland 2013 
graph of bounded degree $\Delta$ is at most $O\left(c^{\Delta}\right)$. In the same paper, Keszegh et al. establish a $3 \Delta-6$ lower bound for the plane slope number of the plane graphs of maximum degree at most $\Delta$, which motivates additional research on reducing the gap between upper and lower bound. The question is studied by Jelínek et al. [5] who prove that the plane slope number of plane partial 3-trees is $O\left(\Delta^{5}\right)$. Also Kant, Dujmović et al., Mondal et al. independently show that the plane slope number of cubic 3-connected plane graphs is six [26]9], whereas the slope number (i.e., when the drawings may contain edge crossings) of cubic graphs is four [10].

In this paper we prove tight bounds (up to a small multiplicative or additive constant) for the plane and the planar slope numbers of planar 2-trees of bounded degree. Our results extend previous papers concerning the planar and plane slope numbers of proper subfamilies of the partial 2-trees. Namely, Jelínek et al. [5] prove that the planar slope number of series-parallel graphs with maximum degree three is at most three. Knauer et al. [8] show that the plane slope number of outerplane graphs with maximum degree $\Delta \geq 4$ is at most $\Delta-1$ and that $\Delta-1$ slopes are sometimes necessary. As a byproduct of our techniques, we answer a long standing open problem by Garg and Tamassia [3], who ask whether $\Omega\left(\frac{1}{\Delta^{2}}\right)$ is a tight lower bound on the angular resolution of seriesparallel graphs of degree $\Delta$ (i.e. they ask whether these graphs admit planar straightline drawings where minimum angle between any two consecutive edges is $\Omega\left(\frac{1}{\Delta^{2}}\right)$ ). More precisely, our results can be listed as follows.

- We prove that the planar slope number of a partial 2-tree of maximum degree $\Delta$ is at most $2 \Delta$ and there exist partial 2-trees whose planar slope number is at least $\Delta$ if $\Delta$ is odd and at least $\Delta+1$ if $\Delta$ is even (Section 3 ).

- We prove that the plane slope number of a plane partial 2-tree of maximum degree $\Delta$ is at most $3 \Delta$ and there exist plane 2-trees whose plane slope number is at least $3 \Delta-3$ if $\Delta$ is even and at least $3 \Delta-4$ if $\Delta$ is odd (Section 4 ).

- We show that a partial 2-tree $G$ of maximum degree $\Delta$ admits a planar straight-line drawing with angular resolution $\frac{\pi}{2 \Delta}$. If $G$ is a plane graph, a plane straight-line drawing of $G$ exists whose angular resolution is $\frac{\pi}{3 \Delta}$ (Section 5). The previously best known bound was $\frac{1}{48 \pi \Delta^{2}}$, established by varying the input embedding [3].

\section{Decomposition Trees and Universal Slope Sets}

In this section we recall some known concepts. Throughout the paper "drawing" means "planar straight-line drawing"; "plane drawing" means "plane straight-line drawing".

SPQ Trees and Block-cut Vertex Trees. Let $G$ be a 2-connected graph. A separation pair is a pair of vertices whose removal disconnects $G$. A split pair of $G$ is either a separation pair or a pair of adjacent vertices. A split component of a split pair $\{u, v\}$ is either an edge $(u, v)$ or a maximal subgraph $G_{u v}$ of $G$ such that $\{u, v\}$ is not a split pair of $G_{u v}$. We call vertices $u$ and $v$ the poles of $G_{u v}$. Note that a split component of $G$ need no t be 2-connected.

A 2-connected series-parallel graph is recursively defined as follows. A simple cycle with three edges is a 2-connected series-parallel graph. The graph obtained by 
replacing an edge of a 2-connected series-parallel graph with a path is a 2-connected series-parallel graph. The graph obtained by adding an edge between the vertices of a nonadjacent separation pair $\{u, v\}$ of a 2-connected series-parallel graph is a 2-connected series-parallel graph. Let $G$ be a 2-connected series-parallel graph. An $S P Q$-tree $T$ of $G$ is a rooted tree describing a recursive decomposition of $G$ into its split components. The nodes of $T$ are of three types: $S, P$, or $Q$. Each node $\mu$ has an associated graph called the skeleton of $\mu$ and denoted by skeleton $(\mu)$. Starting from a split pair $\{s, t\}$ of $G, T$ recursively describes the split components of $G$ as follows. The root of $T$ is a $P$-node corresponding to $G$; its skeleton is defined as in the "Parallel case" below.

- Base case: The split component $H$ is an edge. Then $H$ corresponds to a $Q$-node of $T$ whose skeleton is this edge. The $Q$-nodes are the leaves of $T$.

- Series case: The split component $H$ is a 1-connected graph with split components $H_{1}, \ldots H_{k}(k \geq 2)$ and cut vertices $c_{i}=H_{i} \cap H_{i+1}$. Then $H$ corresponds to an $S$-node $\mu$ of $T$. The graph skeleton $(\mu)$ is a chain $e_{1}, \ldots, e_{k}$ of edges such that $e_{i}=\left(c_{i-1}, c_{i}\right)$, where $c_{0}=s$ and $c_{k}=t$. The children of $\mu$ are the roots of the $S P Q$-trees of $H_{1}, \ldots, H_{k}$.

- Parallel case: Otherwise, the split component $H$ is 2-connected and its split components are $H_{1}, \ldots, H_{k}(k \geq 2)$. Then $H$ has skeleton $(\mu)$ consisting of a set of parallel edges $e_{1}, \ldots, e_{k}$ between $s$ and $t$, one for each $H_{i}$. The children of $\mu$ are the roots of the $S P Q$-trees of $H_{1}, \ldots, H_{k}$.

Figure 1 a) and (b) show a 2-connected series-parallel graph and its $S P Q$-tree, which is uniquely determined by the choice of the initial split pair. Note that no $P$-node ( $S$-node) has a $P$-node ( $S$-node) as a child. Let $T$ be an $S P Q$-tree of a 2-connected series-parallel graph $G$ and let $\mu$ be a node of $T$. The pertinent graph of $\mu$ is the subgraph of $G$ whose $S P Q$-tree is the subtree of $T$ rooted at $\mu$, as shown in Figure 1 (c). The virtual edge of $\mu$ is an edge in the skeleton of the parent of $\mu$ that represents the pertinent graph of $\mu$. Hence for every internal (i.e., non- $Q$ ) node $\mu$ in $T$, each edge in skeleton $(\mu)$ is a virtual edge of some child of $\mu$.

If $\mu$ is $P$-node, then we associate with $\mu$ another graph $\operatorname{frame}(\mu)$, called the frame of $\mu$, which is formed by replacing each edge $e$ in skeleton $(\mu)$ with the skeleton of the child node whose virtual edge is $e$, as shown in Figure 1(d). Every vertex in a frame corresponds to a unique vertex of $G$. Given a vertex $v$ of $G$, the first frame of $v$ is the frame that is closest to the root of $T$ and contains $v$. For any split pair $\{u, v\}$ in a 2-connected series-parallel graph $G$ with $n$ vertices, an $S P Q$-tree having $\{u, v\}$ as reference pair can be computed in $O(n)$ time [4].

A graph $G$ is a partial 2-tree (or, has tree-width at most 2) if and only if each 2connected component of $G$ is either series-parallel or consists of a single edge. Let $G$ be a 1-connected graph. The block-cut vertex tree of $G$, denoted by $B C$-tree, is a graph with vertex set $B \cup C$ such that $B$ consists of one vertex for each block (maximal 2connected subgraph) of $G$ and $C$ consists of one vertex for each cut vertex of $G$. There is an edge from $b \in B$ to $c \in C$ in the $B C$-tree if and only if the vertex of $G$ represented by $c$ belongs to the block represented by $b$.

Universal Slope Sets and Free Wedges. Let $G$ be a graph with vertex set $V$. For a vertex $v \in V$, we denote the degree of $v$ by $\delta(v)$. Hence the maximum degree of $G$ is 


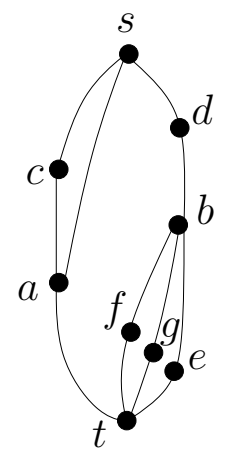

(a)

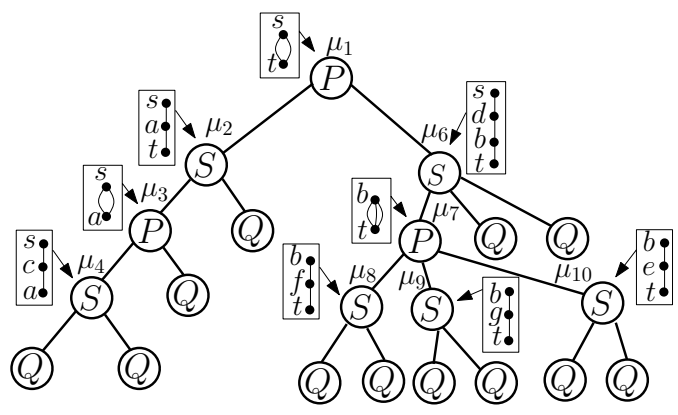

(b)

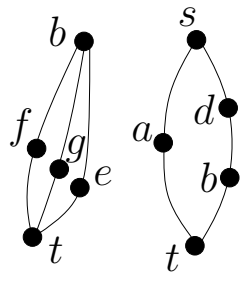

(c)

Fig. 1. (a) A 2-connected series-parallel graph $G$. (b) A $S P Q$-tree of $G$, where the internal nodes are labeled with $\mu_{1}, \ldots, \mu_{10}$. The skeleton of $\mu_{i}, 1 \leq i \leq 10$, is drawn in the box associated with $\mu_{i}$. (c) The pertinent graph of $\mu_{7}$. (d) The frame graph of $\mu_{1}$.

$\Delta=\max _{v \in V} \delta(v)$. The excess of $v$ is $\epsilon(v)=\Delta-\delta(v)$. If $H$ is a subgraph of $G, \delta_{H}(v)$ and $\epsilon_{H}(v)$ are the restrictions of $\delta(v)$ and $\epsilon(v)$ to $H$.

A set $S$ of slopes is universal for a family of planar graphs $\mathcal{G}$ if every graph $G \in \mathcal{G}$ admits a planar straight-line drawing such that the slope of every edge in the drawing belongs to $S$. We consider universal slope sets defined as follows.

Definition 1. Given a positive integer $k \geq 2$, let $\alpha=\frac{\pi}{2 k}$. Define $S_{k}$ to be the set of slopes $i \cdot \alpha$, for $0 \leq i \leq 2 k-1$.

We prove the upper bounds on plane and planar slope numbers showing that there is a value of $k$ depending on $\Delta$ such that $S_{k}$ is universal for the partial 2-trees. In our constructions, we guarantee that some wedge shaped regions of the plane can be used for recursive drawing. In particular, for any $r>0$, point $p \in \mathbb{R}^{2}$, and angle $\phi$, a $\phi$ wedge at $p$ of radius $r$ is a sector of angular measure $\phi$ in the disk of radius $r$ centered at $p$. For convenience, we will often omit reference to $r$, since any suitably small value of $r$ suffices for our purposes. Let $v$ be a vertex in some planar straight-line drawing $\Gamma$. A wedge with its apex at $v$ in $\Gamma$ is a free wedge at $v$ if the wedge intersects the drawing $\Gamma$ only at $v$. The angular measure of our free wedges will depend on the degree and excess of the corresponding vertices.

\section{Slope Number of Partial 2-trees}

In this section we present upper and lower bounds on the planar slope number of partial 2 -trees of maximum degree $\Delta$. We start by studying 2-connected series-parallel graphs (Section 3.1) and then we extend the study to all partial 2-trees (Section 3.2).

\subsection{2-connected Series-parallel Graphs}

In this section we show that $S_{\Delta}$ is a universal slope set for the family of 2-connected series-parallel graphs with maximum degree $\Delta$. 
Lemma 1. Let $\mathcal{G}$ be the family of 2-connected series-parallel graphs having maximum degree at most $\Delta$. Then $S_{\Delta}$ is universal for $\mathcal{G}$.

Proof. The argument is based on a construction that recursively computes a drawing of $G$; the proof is by induction on the number of $P$-nodes in an $S P Q$-tree of $G$. Let $T$ be an $S P Q$-tree of $G$ having split pair $\{s, t\}$ associated with its root and let $m \in S_{\Delta}$. Since $G$ is 2 -connected, $T$ must have at least one $P$-node, e.g., the root of $T$. We show that $G$ admits a drawing $\Gamma$ using only slopes from $S_{\Delta}$ that satisfies the following properties.

(1) Graph $G$ is drawn within a triangle $\triangle a b c$ having $\angle b a c=(\delta(s)-0.5) \alpha$ and $\angle a b c=$ $(\delta(t)-0.5) \alpha$ (i.e. every edge is either in the interior or on the boundary of $\triangle a b c$ ).

(2) Vertices $s$ and $t$ are located at $a$ and $b$, respectively.

(3) Segment $\overline{a b}$ has slope $m$.

(4) The edges incident to $s$ are drawn using consecutive slopes of $S_{\Delta}$, as are the edges incident to $t$.

(5) At each vertex $v \notin\{s, t\}$ in the drawing of $G$, there is a free $\epsilon(v) \alpha$-wedge at $v$ contained in $\triangle a b c$.

Let $a$ and $b$ be two distinct points on a line of slope $m \in S_{\Delta}$, and let $c$ be the point of intersection of the lines through $a$ and $b$ having slopes $m+(\delta(s)-0.5) \alpha$ and $m-(\delta(t)-0.5) \alpha$, respectively.

Base Case: Assume that $T$ has a single $P$-node, which must be the root of $T$ since $G$ is 2 -connected. The frame of the $P$-node consists of a set of paths of length at least 1 (and at most one path of length 1 ). We draw $s$ and $t$ on $a$ and $b$, respectively. We draw one of these paths between $s$ and $t$ along the segment $a b$ with slope $m$ (if there is a path of length one, then we draw that path along $a b$; otherwise, any of the paths can be used). The remaining paths are drawn inside $\triangle a b c$ using slopes $m+i \alpha, i=1, \ldots, \delta(s)-1$ at $a$ and slopes $m-i \alpha, i=1, \ldots, \delta(t)-1$ at $b$ for the edges incident to $s$ and $t$, respectively; we use slope $m$ for all other edges. See Figure 2 (a) for an example.

Let $\Gamma$ be the computed drawing; by construction, $\Gamma$ is crossing-free and it only uses slopes from $S_{\Delta}$. Also, the paths from $s$ to $t$ lie within $\triangle a b c$ and for each vertex $v \notin\{s, t\}$ there is an empty wedge of angle at least $\epsilon(v) \alpha$ with its apex at $v$ that is completely contained within $\triangle a b c$; this wedge is in the " $c$ side" of the path containing $v$, i.e., in the half-plane defined by the line through $a, b$ and containing $c$. Hence, $\Gamma$ satisfies all invariant Properties (1)-(5).

Induction Step: Suppose now that any 2-connected series-parallel graph having at most $j P$-nodes in some $S P Q$-tree admits a drawing that only uses slopes from $S_{\Delta}$ and that satisfies Properties (1)-(5). Let $G$ be a 2-connected series-parallel graph having $j+1 P$-nodes in some $S P Q$-tree $T$. As above, the root of $T$ is a $P$-node and its frame consists of a set of paths $\Pi_{1}, \ldots, \Pi_{k}$ of length at least 1 . We will draw them in a fashion similar to the base case but with one important difference: we do not use consecutive slopes for the edges of the paths incident to $s$ and $t$, but we leave room for the (recursive) drawings of the pertinent graphs associated with each virtual edge incident to $s$ or $t$.

To do this, for each $i=1, \ldots, k$, let $e_{i}$ be the virtual edge incident to $s$ in $\Pi_{i}$ and let $\mu_{e_{i}}$ be the node of $T$ corresponding to the virtual edge $e_{i}$ (note that $\mu_{e_{i}}$ is either a $P$ - or $Q$-node of $T$ ). Further, let $\delta_{e_{i}}(s)$ be the degree of $s$ in the pertinent graph of $\mu_{e_{i}}$. Then $e_{1}$ is drawn using slope $m$, and for each $i>1, e_{i}$ is drawn using 


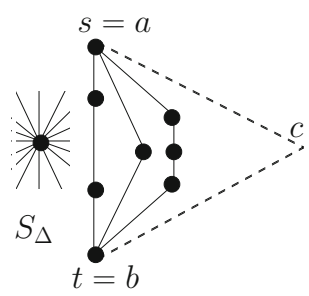

(a)

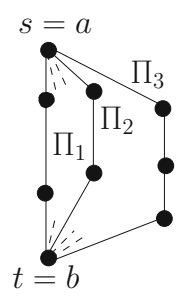

(b)

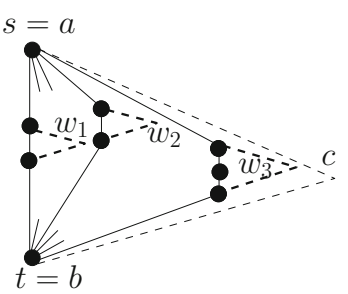

(c)

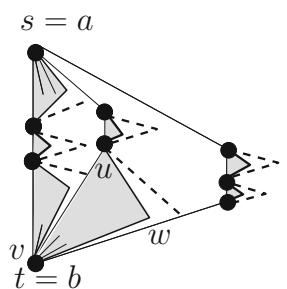

(d)

Fig. 2. (a) An example drawing for Base case, where $\delta(s)=\delta(t)=3$ and $\Delta=4$. (b) Illustration for Inductive step, where $\delta_{e_{1}}(s)=3, \delta_{e_{2}}(s)=1, \delta_{e_{1}}(t)=2$, and $\delta_{e_{2}}(t)=3$. (c) Illustration for $u_{i} v_{i} w_{i}$ in dashed line, and (d) recursive construction.

slope slope $\left(e_{i-1}\right)+\delta_{e_{i-1}}(s) \alpha$. The edges of $\Pi_{i}$ incident to $t$ are positioned similarly, beginning with slope $m$ but decreasing the slopes as we move from one path to the next. See Figure2 (b) for an example.

For every subpath $\Pi_{i} \backslash\{s, t\}$ with at least two vertices $(1 \leq i \leq k)$, we draw the subpath using a sufficiently small line segment as follows. Let $u_{i}, v_{i}$ be the endvertices of that subpath $\Pi_{i} \backslash\{s, t\}$. Let $w_{i}$ be the point of intersection of the half-lines from $u_{i}$ and $v_{i}$ having slopes slope $\left(u_{i} v_{i}\right)+(\Delta-0.5) \alpha$ and slope $\left(u_{i} v_{i}\right)-(\Delta-0.5) \alpha$, respectively. We draw the paths such that $\triangle u_{i} v_{i} w_{i}$ lies in the region bounded by $\Pi_{i}$ and $\Pi_{i+1}$. If $i+1<k$, then $u_{i} v_{i} w_{i}$ lies within $\triangle a b c$. An example is illustrated in Figure 2 (c) in dashed line.

Now that the frame of the root of $T$ has been drawn, let $e=u v$ be a (drawn) virtual edge of a path $\Pi_{i}$ of the frame and let $\mu_{e}$ be its corresponding $P$ - or $Q$-node in $T$. Let $w$ be the point of intersection of the lines from $u$ and $v$ having slopes slope $(e)+$ $\left(\delta_{e}(u)-0.5\right) \alpha$ and slope $(e)-\left(\delta_{e}(v)-0.5\right) \alpha$, respectively. We recursively draw the pertinent graph of $\mu_{e}$ within $\triangle u v w$. If $\{u, v\} \cap\{s, t\}=\phi$, then $\triangle u v w$ is contained in $\triangle u_{i} v_{i} w_{i}$, which by construction does not intersect any part of the already drawn edges. If $e$ is incident to $s$ or $t$ (i.e. either $u=s$ or $v=t$ ), then $\triangle u v w$ does not intersect the edge $e^{\prime}$ of $\Pi_{i+1}$ incident to $s$ or $t$, because by construction, the slope of $e^{\prime}$ is slope $(e)+\delta_{e}(s) \alpha$. Finally, observe that $\triangle u v w$ does not intersect any other triangle that contains the drawing of a pertinent graph associated with a node of $T$ which is not in the subtree rooted at $\mu_{e}$. See Figure2 d) for an illustration.

The observations above, together with the fact that the drawing of the frame graph of the root is crossing-free and that it satisfies Properties (1)-(4), imply that $G$ admits a drawing that only uses slopes from $S_{\Delta}$ satisfying Properties (1)-(4). To see that Property (5) is also satisfied, note that each path $\Pi_{i}$ is drawn as a convex (or linear, for $i=1$ ) chain. Thus at each vertex $v \notin\{s, t\}$ has an angle of at least $\pi$ between its two consecutive (virtual) edges in its first frame. The drawing of $G$ uses two consecutive sets of slopes at $v$, since the pertinent graph for the two nodes corresponding to each of those two virtual edges is drawn using consecutive slopes. This leaves a free wedge of angular measure $\pi-\delta(v) \alpha=2 \Delta \alpha-\delta(v) \alpha=\Delta \alpha+\epsilon(v) \alpha>\epsilon(v) \alpha$ at $v$, establishing Property (5). 


\subsection{Partial 2-trees}

In this section we extend the result of Lemma 1 to partial 2-trees of maximum degree $\Delta$ by proving that $S_{\Delta}$ is universal for these graphs. We shall focus on connected partial 2-trees, since every connected components can be drawn independently of the others.

Lemma 2. Let $\mathcal{G}$ be the family of partial 2-trees having maximum degree at most $\Delta$. Then $S_{\Delta}$ is universal for $\mathcal{G}$.

Proof. We assume that $G$ is connected and has at least one edge. Let $T$ be the blockcutvertex tree of $G$ (see Figures 3(a)-(b)). We build the desired drawing of $G$ by drawing subgraphs of $G$ corresponding to subtrees of $T$, starting with the leaves of $T$. The drawing of $G$ produced will have the following properties:

(a) For some split pair $\{s, t\}$ of $G, G$ is drawn inside a $\delta(s) \alpha$-wedge with apex $s$.

(b) $s$ and $t$ are located on a line of slope $m \in S_{\Delta}$.

(c) The edges incident to $s$ are drawn using consecutive slopes of $S_{\Delta}$, as are the edges incident to $t$.

(d) The wedge of Property (a) is bounded by rays from $s$ in directions $m-0.5 \alpha$ and $m+(\delta(s)-0.5) \alpha$.

If $G$ is 2-connected, then Lemma 1 establishes the existence of a drawing with the desired properties: Property (b) follows obviously from Property (1) of Lemma 1 Property (c) from Property (3) of Lemma 1, and properties (a) and (d) from properties (1), (2) and (4) of Lemma1.

Otherwise, let $s$ be a cut vertex of $G$ and choose $s$ as the root of $T$. The parent of each block vertex $B$ of $T$ is a cut vertex $s_{B}$ of $G$. For each such block $B$, choose a vertex $t_{B}$ in $B$ such that $\left\{s_{B}, t_{B}\right\}$ is a split pair for $B$. Each leaf of $T$ is a block vertex $B$ and by Lemma1 it can be drawn with split pair $\left\{s_{B}, t_{B}\right\}$ inside a wedge with apex $s_{B}$ and angular measure $\delta_{B}\left(s_{B}\right) \alpha$ so that properties (a)-(d) hold.

Assume now that $x$ is a vertex of $T$ for which all subgraphs of $T$ have been drawn respecting properties (a)-(d). Then $x$ represents either a block of $G$ or a cut vertex of $G$. If $x$ represents a cut vertex $v$ of $G$, then let $T_{1}, \ldots, T_{k}$ represent the subtrees of $x$. Let $G_{v}$ be the subgraph of $G$ corresponding to the subtree of $T$ with root $v$. For each of the trees $T_{i}, v$ is at the apex of the $\delta_{T_{i}}(v) \alpha$-wedge in which the subgraph $G_{i}$ of $G$ corresponding to $T_{i}$ has been drawn. These wedges can all be rotated about $v$ so that they use consecutive slopes in $S_{\Delta}$, as shown in Figure 3 (c). Thus the subgraph of $G$ corresponding to the union of $T_{1}, \ldots, T_{k}$ has been drawn in a wedge of angle $\delta_{G_{1}}(v) \alpha+\ldots+\delta_{G_{k}}(v) \alpha=\delta_{G_{v}}(v) \alpha$ with apex $v$ such that properties (a)-(d) are satisfied. Note that by Property (a), the drawing of $G_{v}$ can be made small enough to lie completely inside the free wedge and so does not intersect any other portion of the drawing.

Suppose now that $x$ represents a block $B$ of $G$. The children of $B$ in $T$ represent the cut vertices of $G$ that belong to $B$; let $v$ be one of the child cut vertices of $B$. Draw $B$ in the manner described by Lemma 1 We consider two cases: $v \neq t_{B}$ and $v=t_{B}$ (note that $s_{B}$ is the parent cut vertex of $B$, which is handled by the previous case). 


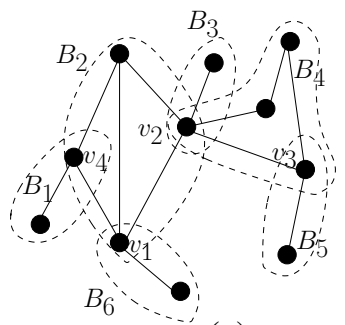

(a)

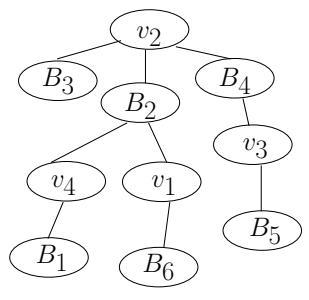

(b)

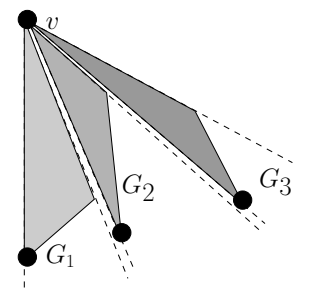

(c)

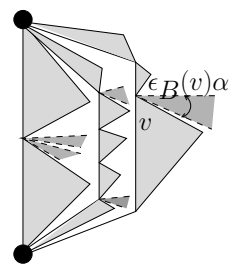

(d)

Fig. 3. (a) A 1-connected graph, and (b) corresponding block-cut vertex tree. (c)-(d) Illustration for the proof of Lemma 2 .

Assume first that $v \neq t_{B}$. Then by Lemma1, there is a free $\epsilon_{B}(v) \alpha$-wedge with apex $v$. Now $\epsilon_{B}(v) \alpha=\left(\Delta-\delta_{B}(v)\right) \alpha>\left(\delta_{G}(v)-\delta_{B}(v)\right) \alpha=\delta_{G_{v}}(v) \alpha$ and so $G_{v}$ can be drawn completely inside the free $\epsilon_{B}(v) \alpha$-wedge with apex $v$ as shown in Figure 3 d).

If $v=t_{B}$, then the drawing of $G_{v}$ already produced can be rotated so that all of the edges in $B$ and of $G_{v}$ adjacent to $t_{B}$ use consecutive (clockwise from the line containing $s_{B}$ and $t_{B}$ ) directions in $S_{\Delta}$ as shown in Figure 3 d). It is an easy observation that the addition of the subgraphs $G_{v}$ for each cut vertex of $B$ into the free wedge preserves properties (a)-(d) of the drawing of $B$ (and of the drawing of $G$ ).

Lemma 3. For any $\Delta>3$, there exists a 2-connected series-parallel graph $G$ of maximum degree $\Delta$ whose planar slope number is at least $\Delta+1$ if $\Delta$ is even and at least $\Delta$ if $\Delta$ is odd.

Proof. Consider the graph $G$ obtained from $K_{2, \Delta-1}$ by adding the edge $(u, v)$ connecting the two vertices $u$ and $v$ of degree $\Delta-1$. Thus $G$ has maximum degree $\Delta$. Now consider any drawing of $G$. At least half of the remaining $\Delta-1$ vertices are on one side of the line determined by the segment representing $u v$ in the drawing. Each of these $\lfloor\Delta / 2\rfloor$ vertices forms a triangle with $u v$ and these triangles are nested. Thus no two of the $2\lfloor\Delta / 2\rfloor+1$ edges in this portion of the drawing of $G$ have the same slope.

The following theorem is an immediate consequence of Lemmas 2 , and 3

Theorem 1. Let $G$ be a partial 2-tree having maximum degree $\Delta$ and let $p s l(G)$ denote the planar slope number of $G$. Then $\operatorname{psl}(G) \leq 2 \Delta$. Also, for every even $\Delta>3$ there exists a partial 2-tree $G$ such that $\operatorname{psl}(G) \geq \Delta+1$ and for every odd $\Delta \geq 3$ there exists a partial 2-tree $G$ such that $\operatorname{psl}(G) \geq \Delta$.

\section{Plane Slope Number of Partial 2-trees}

In this section we show that the plane slope number of 2-connected series-parallel graphs, i.e., when the output drawings respect the input embeddings, is at least $3 \Delta-4$ and at most $3 \Delta$. In fact, we show that $S_{1.5 \Delta}$ is universal for the family of 2-connected series-parallel graphs with fixed embeddings. 
We introduce some additional notation. For an embedded planar 2-connected seriesparallel graph $G$ with poles $s$ and $t$, we call the edge $(s, t)$ (if exists) the central edge of $G$. Since $G$ is 2-connected, the root of its $S P Q$-tree is a $P$-node $\mu$. Observe that each of the edges in skeleton $(\mu)$ corresponds to either the edge $(s, t)$ or a 2-connected series-parallel subgraph of $G$. If the edge $(s, t)$ exists, then we categorize each of those subgraphs as a left or right series-parallel subgraph of $G$ depending on whether it lies to the left or right of the edge $(s, t)$, while walking from $s$ to $t$. By $G_{1}^{-}, \ldots, G_{l}^{-}$, (respectively, $G_{1}^{+}, \ldots, G_{r}^{+}$) we denote the left (respectively, right) series-parallel subgraphs of $G$. If $(s, t)$ does not exist, then we assume that all the series-parallel subgraphs are right series-parallel, i.e., $G_{1}^{+}, \ldots, G_{r}^{+}$. Furthermore, we assume that the subgraphs are ordered as follows: $G_{l}^{-}, \ldots, G_{1}^{-},(s, t), G_{1}^{+}, \ldots, G_{r}^{+}$, reflecting their left to right ordering in the embedding.

Lemma 4. Let $\mathcal{G}$ be the family of plane 2-connected series-parallel graphs of maximum degree at most $\Delta$. Then $S_{1.5 \Delta}$ is universal for $\mathcal{G}$.

Sketch of Proof: Similar to Lemma 1 we employ an induction on the number of $P$ nodes in an $S P Q$-tree $T$ of $G$. Since the proof follows a similar argument, for reasons of space we sketch here the main idea of the proof. Let $\{s, t\}$ be the split pair associated with the root of $T$, and let $\overline{a b}$ be a straight line segment with slope $m$, where $m \in$ $S_{1.5 \Delta}$. We show that $G$ admits a drawing $\Gamma$ using only slopes from $S_{1.5 \Delta}$ such that the following properties hold.

(1) Graph $G$ is drawn within a convex quadrilateral $\square a d b c$ having $\angle d a c=\delta(s) \alpha$ and $\angle d b c=\delta(t) \alpha$ (i.e. every edge is in the interior of $\square a d b c$ ).

(2) Vertices $s$ and $t$ are located at $a$ and $b$, respectively.

(3) Segment $\overline{a b}$ has slope $m$.

(4) The edges incident to $s$ are drawn using consecutive slopes of $S_{1.5 \Delta}$, as are the edges incident to $t$.

(5) At each vertex $v \notin\{s, t\}$ in the drawing of $G$, there are two free $\epsilon(v) \alpha$-wedges at $v$ contained in $\square a d b c$, one in the region between the subgraph $G_{i}^{+}$(or $G_{i}^{-}$) containing $v$ and the previous series-parallel subgraph of $G$ in the ordering, and one in the region between $G_{i}^{+}$(or $G_{i}^{-}$) and the next series-parallel subgraph of $G$ in the ordering.

Base Case: Assume that $T$ has a single $P$-node, which must be the root of $T$ since $G$ is 2 -connected. We draw $s$ and $t$ on $a$ and $b$, respectively. The frame of the $P$-node consists of a set of paths of length at least one (and at most one path of length one). If the central edge exists, then we draw that edge along $\overline{a b}$ (otherwise, we draw the leftmost path along $\overline{a b}$ ). We then draw the paths $\Pi_{i}^{+}, i=1, \ldots, r$ corresponding to each $G_{i}^{+}$ between $a$ and $b$ using consecutive slopes at $s$ and $t$, as in the proof of Lemma 1 . All the remaining paths $\Pi_{i}^{-}, i=1, \ldots, l$ corresponding to $G_{i}^{-}$are drawn symmetrically to the left of segment $\overline{a b}$, as shown in Figure 4 a). While drawing the paths, we maintain the input embedding. To construct the quadrilateral $\square a d b c$, let $d$ be the intersection of the line through $a$ having slope $0.5 \alpha$ plus the slope of the edge of $\Pi_{r}^{+}$incident to $s$ with the line through $b$ having slope $-0.5 \alpha$ plus the slope of the edge of $\Pi_{r}^{+}$incident to $t$. Similarly, let $c$ be the intersection of the line through $a$ having slope $-0.5 \alpha$ plus the 


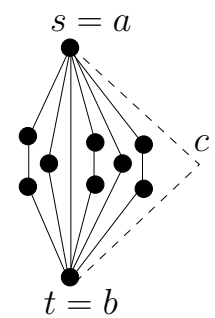

(a)

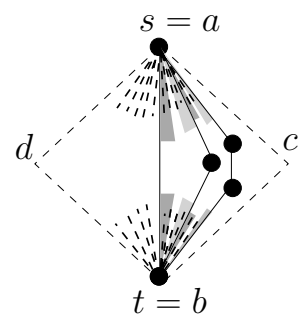

(b)

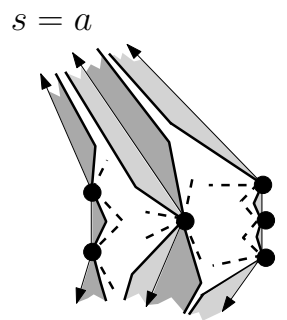

(c)

Fig. 4. (a) Base case. (b) Drawing frame, where the light-gray (respectively, dark-gray) regions correspond to the right (respectively, left) series-parallel subgraphs of the corresponding pertinent graph. (c) Recursive construction.

slope of the edge of $\Pi_{l}^{-}$incident to $s$ with the line through $b$ having slope $0.5 \alpha$ plus the slope of the edge of $\Pi_{l}^{-}$incident to $t$. This convex quadrilateral has angle $\delta(s) \alpha$ at $a$ and $\delta(t) \alpha$ at $b$. See Figure 4(a) for an example.

It is now straightforward to observe that the resulting drawing is planar and satisfies properties (1)-(4). As for Property (5), consider $v \notin\{s, t\}$ in one of the frame paths and its neighbors $u$ and $w$ on that path. Each of the two angles $\angle u v w$ is either non-acute or at least $\pi-(\delta(u)-1) \alpha-(\delta(w)-1) \alpha \geq(\Delta+2) \alpha \geq \epsilon(v) \alpha$, and so the required empty wedges exist at $v$.

Induction Step: In a way similar to the proof of Lemma 1, we first draw the frame of the root of the $S P Q$-tree and then define disjoint convex quadrilaterals for each of the virtual edges. Finally, we recursively compute the drawings of the pertinent graphs inside the corresponding quadrilaterals. The idea is illustrated in Figures $4(b)-(c)$.

By Property (5) of Lemma 4 for every vertex $v \notin\{s, t\}$ in $G$, there is a free wedge on each side of the drawing of the pertinent subgraph $G_{i}^{+}\left(G_{i}^{-}\right)$of $G$ containing $v$. Thus the arguments used in the proof of Lemma 2 can be directly applied in the fixed embedding case to establish the following lemma.

Lemma 5. Let $\mathcal{G}$ be the family of plane partial 2-trees of maximum degree at most $\Delta$. Then $S_{1.5 \Delta}$ is universal for $\mathcal{G}$.

We observe that the $\Delta-1$ lower bound proved by Knauer et al. [8] for the outerplane slope number implies a lower bound for plane slope number of plane partial 2-trees, because outerplane graphs are plane partial 2-trees. The next lemma shows a better lower bound for partial 2-trees.

Lemma 6. For every $\Delta \geq 2$, there exists a plane 2-connected series-parallel graph $G$ of maximum degree $\Delta$ whose plane slope number is at least $3 \Delta-3$ if $\Delta$ is even and at least $3 \Delta-4$ if $\Delta$ is odd.

Proof. Suppose first that $\Delta \geq 2$ is an even number and consider a plane partial 2tree $G$ defined as follows. The external face of $G$ is a 3-cycle with vertices $a, b, c$. In its interior there are $\Delta / 2-1$ paths of length two connecting each pair from $\{a, b, c\}$. 
The external face of every plane drawing $\Gamma$ of $G$ is a triangle $\triangle a b c$ that contains the paths of length two in its interior. From elementary geometry, no two edges of $\Gamma$ can have common slope, and hence the graph, which has $3 \Delta-3$ edges, has plane slope number at least $3 \Delta-3$. Suppose now that $\Delta$ is odd and let $\Delta^{\prime}=\Delta+1$. Construct a graph $G^{\prime}$ of maximum degree $\Delta^{\prime}$ as described above. Now remove one of those paths of length 2 connecting $a$ and $b$ and one connecting $c$ and $b$ from $G^{\prime}$. This new graph, $G$, has maximum degree $\Delta=\Delta^{\prime}-1$ and it requires a different slope for each edge. Since we deleted four edges from $G^{\prime}, G$ has $\left(3 \Delta^{\prime}-3\right)-4=3 \Delta-4$ edges. Thus $3 \Delta-4$ slopes are required, and the result is established.

Lemmas 4 and 6 imply the following.

Theorem 2. Let $G$ be a plane partial 2-tree having maximum degree $\Delta$ and let epsl $(G)$ denote the plane slope number of $G$. Then epsl $(G) \leq 3 \Delta$. Also, for every even $\Delta>3$ there exists a plane partial 2-tree $G$ such that epsl $(G) \geq 3 \Delta-3$ and for every odd $\Delta \geq 3$ there exists a plane partial 2-trees $G$ such that epsl $(G) \geq 3 \Delta-4$.

\section{Angular Resolution}

The angular resolution of a planar straight-line drawing is the minimum angle between any two edges incident to a common vertex. The angular resolution of a planar graph $G$ is the maximum angular resolution over all possible drawings of $G$.

Malitz and Papakostas [11] show that the angular resolution of a planar graph of maximum degree $\Delta$ is $\Omega\left(\frac{1}{7 \Delta}\right)$. Garg and Tamassia [3] show that there exist planar 3trees of maximum degree $\Delta$ that require angular resolution $O\left(\sqrt{\frac{\log \Delta}{\Delta^{3}}}\right)$. They also show that for a subfamily of the partial 2-trees of bounded degree, namely the series-parallel graphs of maximum degree at most $\Delta$, the angular resolution is at least $\frac{1}{48 \pi \Delta^{2}}$. Their drawing technique does not apply to plane graphs since it may vary a given combinatorial embedding. Garg and Tamassia leave as open the problem about whether $\Omega\left(\frac{1}{\Delta^{2}}\right)$ is a tight lower bound for the angular resolution of the series-parallel graphs.

An implication of the drawing techniques of the previous sections of this paper is that the angular resolution of partial 2-trees (and thus also of series-parallel graphs) is in fact $\Omega\left(\frac{1}{\Delta}\right)$. Namely, the constructions of Lemmas 2 and 4 either use the universal set $S_{\Delta}$ or the universal set $S_{1.5 \Delta}$ which consist of equally spaced slopes; therefore, the minimum angle between any two edges sharing a common end-vertex is either $\frac{\pi}{2 \Delta}$ in the variable embedding setting or it is $\frac{\pi}{3 \Delta}$ in the fixed embedding setting. Therefore:

Theorem 3. A partial 2-tree of maximum degree $\Delta$ admits a planar straight-line drawing with angular resolution $\frac{\pi}{2 \Delta}$. A plane partial 2-tree of maximum degree $\Delta$ admits a planar straight-line drawing with angular resolution $\frac{\pi}{2 \Delta}$.

\section{Open Problems}

An interesting research direction is to study the trade-off between the slope number and the area requirement of planar graphs. Similar studies have been carried out for the 
angular resolution and the area requirements of planar graphs having maximum degree at most $\Delta$ (see, e.g., [3]).

Another fascinating open problem is to close the gap between upper and lower bounds on the planar/plane slope number of planar/plane graphs of bounded degree (see [7]). This would be interesting even restricted to partial 3-trees (see [5]).

Acknowledgments. Part of this research took place during the 12th INRIA-McGillVictoria Workshop on Computational Geometry, held in February 2-8, 2013, at the Bellairs Research Institute of McGill University. We thank the organizers and the participants for the opportunity and the useful discussions. Special thanks go to Zahed Rahmati for early thoughtful insights on the problems of this paper.

\section{References}

1. Di Battista, G., Eades, P., Tamassia, R., Tollis, I.G.: Graph Drawing. Prentice- Hall, Englewood Cliffs (1999)

2. Dujmović, V., Eppstein, D., Suderman, M., Wood, D.R.: Drawings of planar graphs with few slopes and segments. Computational Geometry 38(3), 194-212 (2007)

3. Garg, A., Tamassia, R.: Planar drawings and angular resolution: Algorithms and bounds. In: van Leeuwen, J. (ed.) ESA 1994. LNCS, vol. 855, pp. 12-23. Springer, Heidelberg (1994)

4. Gutwenger, C., Mutzel, P.: A linear time implementation of SPQR-trees. In: Marks, J. (ed.) GD 2000. LNCS, vol. 1984, pp. 77-90. Springer, Heidelberg (2001)

5. Jelínek, V., Jelínková, E., Kratochvíl, J., Lidický, B., Tesar, M., Vyskocil, T.: The planar slope number of planar partial 3-trees of bounded degree. Graphs and Combinatorics 29(4), 981-1005 (2013)

6. Kant, G.: Hexagonal grid drawings. In: Mayr, E.W. (ed.) WG 1992. LNCS, vol. 657, pp. 263-276. Springer, Heidelberg (1993)

7. Keszegh, B., Pach, J., Pálvölgyi, D.: Drawing planar graphs of bounded degree with few slopes. SIAM J. Discrete Math. 27(2), 1171-1183 (2013)

8. Knauer, K., Micek, P., Walczak, B.: Outerplanar graph drawings with few slopes. In: Gudmundsson, J., Mestre, J., Viglas, T. (eds.) COCOON 2012. LNCS, vol. 7434, pp. 323-334. Springer, Heidelberg (2012)

9. Mondal, D., Nishat, R.I., Biswas, S., Rahman, M.S.: Minimum-segment convex drawings of 3-connected cubic plane graphs. Journal of Combinatorial Optimization 25(3), 460-480 (2013)

10. Mukkamala, P., Pálvölgyi, D.: Drawing cubic graphs with the four basic slopes. In: Speckmann, B. (ed.) GD 2011. LNCS, vol. 7034, pp. 254-265. Springer, Heidelberg (2011)

11. Papakostas, A., Tollis, I.G.: Improved algorithms and bounds for orthogonal drawings. In: Tamassia, R., Tollis, I.G. (eds.) GD 1994. LNCS, vol. 894, pp. 40-51. Springer, Heidelberg (1995) 